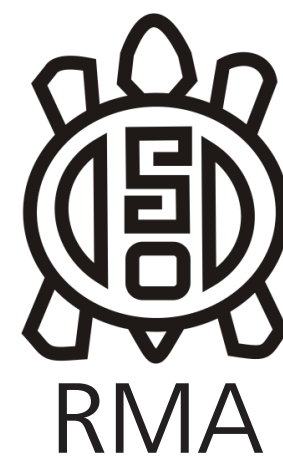

Dossier

Wichí: El mundo

\title{
Asterismos, cielo y ciclos temporales entre los wichís
}

\author{
Asterisms, sky and time cycles among the Wichi
}

\author{
Cecilia Paula Gómez*
}

Resumen

El objetivo del presente artículo es brindar una descripción preliminar del ámbito celeste y de algunos de los ciclos temporales que, desde la óptica wichí, también se asocian al cielo. Para este primer abordaje se parte de la prospección etnográfica que se comenzó en un barrio de la ciudad de Ingeniero Juárez (provincia de Formosa, Argentina) que está habitado mayormente por wichís. Además, recaba y sistematiza la información bibliográfica disponible respecto de las poblaciones wichis que habitan a lo largo de la zona chaqueña. Finalmente, se revisan una serie de líneas de trabajo propuestas con antelación, que estimamos nos permitirán explorar a la sociedad wichí a partir una óptica que tiene como punto de partida las lecturas que hacen del ámbito celeste.

Palabras clave: Wichi; Gran chaco; Cielo; Asterismos; Ciclos temporales.

\begin{abstract}
This article aims to provide a preliminary description of the celestial domain and some of the time cycles which, from the Wichi point of view, are associated with the sky. For this first approach, the starting point will be the ethnographic research initially carried out in a neighborhood of the Ingeniero Juárez city (Formosa, Argentina) which is largely inhabited by Wichi people. Also, available bibliographic information about Wichi communities inhabiting the Chaco territory is taken into account. Finally, there is a review of a series of work lines previously proposed which we assume will allow us to study the Wichi society taking the readings they make of the sky as a starting point.
\end{abstract}

Keywords: Wichi; Gran chaco; Sky; Asterisms; Time cycles.

\section{Introducción}

A partir del año 2005 iniciamos nuestra investigación sobre astronomía cultural ${ }^{1}$ tomando en cuenta sobre todo la información bibliográfica disponible sobre los wichís. Posteriormente, continuamos una investigación etnográfica en el campo con los tobas que habitan en el oeste formoseño, también llamados "toba pilagás". Pero, hacia mediados del 2016, más precisamente en el mes de julio, realizamos una primera prospección etnográfica con los wichís que actualmente habitan en el Barrio Viejo de Ingeniero Juárez. ${ }^{2}$ Luego de una semana en la zona logramos comenzar a trabajar con una mujer mayor que accedió a hablar sobre la temática que planteábamos y era maestra bilingüe (MEMA: Maestra Especial Modalidad Aborigen). Ella se emparentaba con los wichís

\footnotetext{
1 A grandes rasgos, podemos decir que este campo de investigación indaga sobre el conocimiento y la relación que distintos grupos humanos establecen con el cielo y con todo lo que consideren que se halla relacionado con él (ver p. ej. Aveni, 1991; Belmonte, 1999; Iwaniszski, 1994; Ruggles \& Sanders, 1993).

2 Las coordenadas geográficas de la ciudad de Ingeniero Juárez es de $23^{\circ} 54^{\prime} \mathrm{S}$ y $61^{\circ} 51^{\prime} \mathrm{O}$.
}

bermejeños, habiendo sido su padre de Rivadavia Banda Sur (provincia de Salta), y su madre "también del Bermejo, de Formosa". En un principio nos pidió algo más de tiempo para volver a indagar con ella sobre el imaginario relacionado con el cielo, debía tratar de recordar: puesto que hoy en día el cielo no parece tener tanta presencia cotidiana en la ciudad, no es tan necesario como antaño recordar sus señales. Pero luego, poco a poco, nos fue contando y mostrando lo que recordaba. Teniendo en cuenta que para este artículo hemos trabajado con una mujer mayor que ha oficiado de "colaborador clave", se podría plantear que la visión del cielo aquí propuesta es necesariamente parcial. Este trabajo es sólo un puntapié inicial a partir del que se propone una primera puesta al día de los conocimientos celestes wichís, poniendo atención a los cambios que se perciben entre lo plasmado en la bibliografía y lo que plantea una mujer mayor vinculada al ámbito de la enseñanza formal. Por lo tanto, aquí sólo se presenta un primer atisbo de lo que sucede en relación con el cielo en un contexto urbano. A la vez, este bosquejo sólo puede plantearse cabalmente en conjunto con una exhaustiva revisión bibliográfica que ha de completar y contextualizar lo expresado aquí en el 
conjunto mayor de lo trabajado con antelación por otros investigadores entre los wichís.

El presente trabajo, en consecuencia, toma tanto la información recabada en el campo como la proveniente de la bibliografía etnográfica, indagando sobre todo aquello que se relacione con el conocimiento wichí del cielo. Se hará una descripción preliminar de los asterismos y de algunas de las significaciones que culturalmente se asocian con ellos. Finalmente, se ahondará en algunas interpretaciones de los mitos asociados con los asterismos que desde el plano celeste parecen interpelar a los wichís -relacionadas, en particular, con el proceso de autocontrol personal y colectivo que busca desarrollar la "buena voluntad" wichí (Palmer 2005).

\section{Los momentos del ciclo anual}

Pastor Arenas (2003, pp. 181-196) describe una visión del ciclo anual diferente de la aportada en estas páginas. La versión registrada por el autor para los wichís /hokotas consta de más ciclos que la escrita aquí, que resulta más esquemática y breve. Arenas (2003, p. 182) advierte de la existencia de otra descripción del ciclo anual además de la descripta por él, y que tal como la variación relevada por nosotros se adapta con extraordinaria plasticidad al calendario occidental, agrupando sus ciclos en cuatro secciones. En la versión del ciclo anual trabajada por nosotros, la referencia a asterismos $u$ objetos celestes se reduce principalmente a dos: Wel'a, Luna, y Wän/häj, el suri ${ }^{3}$ celeste. En principio, no se ha recabado aquí aquello que sí se ha descripto entre los wichís del Pilcomayo donde, como escribe Montani $(2017$, p. 63) retomando a Alvarsson (1988, p. 58) y Fock (1982, p. 9), el comienzo del ciclo anual tradicional se produce cuando se comienza a ver a las Pléyades hacia el Este del horizonte celeste, un poco antes del amanecer. A ese momento se suma que se dan los días más cortos y noches más largas del año para el hemisferio Sur, cuando se produce el solsticio de invierno. Según lo relevado por Pérez Diez (1974, p. 118), el asterismo llamado Pätselhay es representado por las Pléyades y estaría señalando la mitad del año, nekchäm chowej. Esta mitad anual wichí estaría marcada por la desaparición y nueva aparición de las Pléyades. ${ }^{4}$

En nuestro primer acercamiento al tema en el campo, la mayor cantidad de referencias para identificar cada ciclo estacional se relaciona a las plantas y los animales característicos. Cada uno de los grandes ciclos contiene

\footnotetext{
${ }^{3}$ El ave a la que refiere el asterismo es el ñandú (Rhea americana).

${ }^{4}$ El mismo autor también refiere que las Pléyades dejan de verse hacia fines de julio (Pérez Diez, 1974, p. 118). Sin embargo, esa afirmación podría derivar de una confusión, puesto que las Pléyades sí pueden ser vistas en esa época del año en la zona del departamento Rivadavia donde el autor realizó su trabajo de campo. Tal vez el autor haga referencia a alguna circunstancia de orden meteorológico o coyuntural que haya impedido su visión. Otra posibilidad sería que los wichís consideren que en esa época Pätselhay deja de verse, aunque las Pléyades sí puedan ser vistas en el cielo de esa zona.
}

en sí secciones más pequeñas que tienen como referencia a Wel'a (Luna). Para estas secciones más pequeñas se presta especial atención al recorrido y cambios de fases que presenta la Luna, haciéndose referencia a lo que la astronomía académica denomina mes sinódico. ${ }^{5}$ Se hacía hincapié en que al sumar las Wel'a (Luna) presentes en cada una de las cuatro grandes secciones en las que se divide el ciclo anual, no se debía superar las doce Lunas. Para explicarnos el porqué de esta suma se especificó lo siguiente: "nosotros decimos lunas, no decimos meses". En base a lo expresado es evidente que wel'a o iwel'a significa tanto Luna como mes. En otro trabajo de mediados de la década de 1970 se hace referencia que el número de lunas que debería tener un ciclo anual es de catorce lunas o wel'as (Pérez Diez, 1974, p. 115). ${ }^{6}$ Esta actual asimilación entre cantidad de lunas y meses englobadas dentro del ciclo anual podría ser otra de las muestras del intento de coordinar el ciclo anual wichí con el del calendario occidental, puesto que se busca que la cantidad de lunas de cada ciclo coexista con los doce meses del año calendario occidental, tratando de que ambos encastren con la mayor exactitud posible, aunque algunas veces el año calendario puede contener por lo menos un ciclo lunar más. Pérez Diez (1974, p. 115) llega a la conclusión de que la identificación entre Luna y mes, probablemente, se relacione a los contactos con el blanco en los ingenios azucareros. En su diccionario de la lengua wichí, Lunt expresa que para referir al mes que viene se puede decir: iwel'a tä tatay alhoho, donde Iwel'a también refiere a Luna. Lo escrito en este párrafo, sin embargo, no indica que los wichís no utilizaran tradicionalmente a la Luna para referir a intervalos de tiempo más largos que los días, más pequeños que las "estaciones" y relacionados con la menstruación.

Respecto de los distintos momentos o "estaciones" en las que se divide el ciclo anual, en nuestro trabajo de campo lo que primero se ha descripto fue el momento de mayor frío: fwuily etil. $^{7}$ El nombre de este ciclo hace referencia a la helada que cae en ese período: fwiyet (helada). En esa instancia es cuando aparece la otra referencia estelar: Wän/häj, el suri celeste que está cercano a la Cruz del Sur, que corresponde y anuncia esta época fría, que es también la de mayor sequía. Para esa misma época, a medida que pasa la noche, hacia el otro lado por el que se pone "el pico" de wänlhäj, el suri, también estará Pätselhay, las Pléyades. Fwuiy'etil tiene sólo dos wel'as, que a partir de nuestra óptica abarcaría los meses de junio

\footnotetext{
${ }^{5}$ Mes sinódico o período sinódico "es el tiempo que transcurre entre dos conjunciones consecutivas de la Luna con el Sol, como se observa desde la Tierra" (Feinstein, 1969, p. 200)

${ }^{6}$ En un esquema que dibuja el autor, se reparte cada una de las Lunas en cinco "estaciones", ese esquema tiene variaciones con el que aquí se describe. Esta situación es lógica, puesto que la percepción y forma de nombrar estas secciones más pequeñas que el ciclo anual, pero más largas que los meses o lunaciones, varía mucho de comunidad en comunidad.

7 "Fwiy'et, pl. fwiy'etil: invierno, tiempo frío" (Lunt, 2016, p. 32).
} 
y julio. Es un momento de escasez ${ }^{8}$, en el que ni siquiera hay gusanos, no hay alimento para los pájaros en los árboles y éstos comienzan a caminar por la tierra. En ese momento el quirquincho (Tolypeutes matacus), la chuña (Chunga burmeisteri) y la charata (Ortalis canicollis) están sin embargo en óptimas condiciones para el consumo, o como se expresó con respecto al primero: "se pone lindo, gordo". También abunda el pescado en el río. Luego viene nawop, ${ }^{9}$ cuando comienza el brote de las primeras hojas y flores, es la época de la miel y abunda el pescado. La duración de nawop es de tres wel'as, lo que correspondería a los meses de agosto, septiembre y octubre. La temporada que sigue es llamada yachep. ${ }^{10}$ Esta parte del ciclo es el momento de abundancia, aparece la iguana (alhe, Tupinambis rufescens), el monte está con muchos frutos y es el tiempo de la cosecha. Yachep es un momento de festejos: "ahí se festeja también el año (...) se festejaba porque dicen que son los meses más lindos del año. Como expreso mi informante: "hay abundante alimento". Esta época tiene cuatro wel'as, que se las hace corresponder a los meses noviembre, diciembre, enero y febrero. El último de los ciclos descriptos es lup, es traducido como "otoño", comienzan las lloviznas y se empieza a pudrir todo lo que hay en el monte, comienza la escasez que se recrudece en fwiyetil, cuando todo se pone más seco y comienza la helada (fwiyet). Lup consta de tres wel'as que se asimilan a los meses de marzo, abril y mayo ${ }^{11}$.

\section{El cielo, objetos celestes y algunos asterismos}

Durante el trabajo etnográfico se recabaron descripciones del ámbito celeste, algunas de ellas coincidentes con lo encontrado en la bibliografía disponible y otras que parecen brindar una mirada distinta. Al hablar sobre el cielo durante el trabajo de campo, lo primero que se relató fue la inversión de los planos celeste y terrestre. El cielo o pule antes estaba abajo, en el lugar que ahora ocupa la tierra. El cielo fue descripto como un lugar brilloso en el que todos hacían sus necesidades, el problema era que ese suelo no las absorbía, de ahí que el lugar se llenaba de excrementos tornándose una superficie muy resbalosa en la que "no se podía trabajar". Por ese motivo, finalmente, se invirtió la ubicación de la tierra y el cielo, ocupando éste último el lugar que tiene hoy: arriba. Los "seres" que vivían allí subieron al cielo, entre ellos: Luna (Wel'a), las estrellas (katetsel) y Sol (Fwala). Los que se quedaron en la tierra ya podían andar por aquí porque la tierra era diferente: aquí ya se podían tirar cosas y al ser absorbidas por la tierra no causaban inconveniente. Al trabajar con la literatura etnográfica, puede notarse

\footnotetext{
${ }^{8}$ De hecho, otro de los nombres de esta época del ciclo anual es "lop", que también significa que hay escasez, sobre todo escasez de carne (Comentario personal Rodrigo Montani).

9 "Nawup: primavera" (Lunt 2016, p. 64). Término vinculado con "Inawo: flor (general)" (Lunt 2016, p. 40).

10 "Yachup: verano, tiempo de fruta" (Lunt 2016, p. 109)

11 Para otras referencias a ciclos anuales wichís ver, Fock, 1982, pp. 8-13; Montani 2017, pp. 62-63; Palmer, 2005, p. 12; Pérez Diez, 1974, pp. $115-119$
}

que anteriormente han sido recogidas otras versiones de este mito sobre la inversión de los planos terrestre y celeste. Una de ellas, por ejemplo, indica que una deidad llamada Nilataj se asqueó de los excrementos que caían al cielo y decidió invertir el orden, colocando a la tierra debajo del cielo (Braunstein, 1974, p. 23). Ya a principios del siglo XX Alfred Métraux (1939, p. 9) recogió una versión similar a las expuestas, en la que se describe un cosmos con por lo menos tres niveles, en el que también el cielo y la superficie terrestre invirtieron sus lugares. El mundo subterráneo es la morada de los muertos, el espacio medio sería la tierra y por encima estaría el cielo. Con respecto al mundo subterráneo, fue descripto por los wichís porque lo visitaban durante sus sueños. Por otro lado, se entiende que cuando sobre la tierra es de noche y están las estrellas, es porque en el mundo de abajo está brillando el Sol. Esta idea es congruente con aquella que explica la "salida" y "entrada" del Sol por un agujero o cavidad que se abre hacia el este y el oeste de la tierra respectivamente. Luego de pasar por una de estas aberturas, el Sol seguiría su trayecto por debajo de la tierra para volver a asomar por la otra abertura hacia la superficie terrestre conocida por los vivos. ${ }^{12}$

Sobre el sexo de los habitantes del cielo nombrados, vimos que la Luna (Wel'a) es pensada en masculino, sobre el Sol (Fwala) no hubo definición y las estrellas (katés) son pensadas en femenino. Respecto de estas últimas, como en otros grupos chaqueños, aquí también se cuenta sobre la llegada de las mujeres desde el cielo. Las mujeres que ahora están en la tierra bajaron del cielo y, al igual que aquellas que quedaron en el cielo, eran estrellas. Sólo para tener una mínima referencia resumiremos aquí la versión recabada durante el trabajo de campo ${ }^{13}$. En la tierra vivían los hombres primigenios que eran animales, quienes iban a mariscar. Estos personajes por la noche traían a su campamento lo que habían cazado, pescado y recolectado, y lo que no llegaban a comer lo dejaban en un encatrado (lekawet). Cuando volvían al monte dejaban todo allí. Era precisamente mientras que estos protohombres se ausentan cuando las protomujeres bajaban del cielo y comían las cosas que habían dejado sobre el encatrado. Los protohombres se dieron cuenta que les faltaban cosas y dejaron un vigilante para saber quién venía a robar. El primero que dejaron fracasó en su empresa, pero el último, el protohombre que era un halcón, logró atrapar a las mujeres que venían por el alimento. Las mujeres que bajaban del cielo eran "todas blancas, usaban ropas blancas, eran hermosas". El ave cortó la escalera por donde bajaban las mujeres y ellas no pudieron volver a subir, "se quedaron muy asustadas y se arrinconaron en una pieza, en la casa". Luego cada protohombre agarró a una mujer, y así se conformaron las

\footnotetext{
${ }^{12}$ Para croquis y otras descripciones sobre la concepción de la tierra y espacios cosmográficos significativos para los wichís ver Dasso, 2018, pp. 141-143; Montani, 2017, pp. 51-53.

13 Durante el trabajo de campo no nos brindaron el nombre o "título" del mito pero, por ejemplo, Alfred Métraux lo ha titulado como: "The first woman" (Métraux, 1939, pp. 49-52).
} 
primeras parejas, que se fueron esparciendo por la tierra y de las que "nacieron los hijos, y así cada uno con su pareja: esos somos nosotros". Por otro lado, las distintas características de las personas actuales se derivan de ciertas características de aquellos protohombres/animales primigenios. Por ejemplo, si alguien tiene mucho pelo es por ser descendientes del quirquincho (Tolypeutes matacus).

Como se dijo más arriba, Luna (Wel'a) es pensada en masculino y el ciclo de cambios que muestra en el cielo (su cambio de forma y movimientos realizados en la "esfera celeste") ayudan a marcar junto con otras señales los segmentos temporales más pequeños dentro de las grandes estaciones en las que los wichís dividen el ciclo anual. Durante el trabajo de campo quedó claro que los aparentes cambios de forma de Luna y sus movimiento en el cielo se secuencian al ritmo del ciclo vital de un ser vivo. Cuando se da la primera aparición de la Luna, aquello que la astronomía académica llama "primer cuarto de ciclo de fases", se piensa como el "brote" de Luna: tälh, "brota". Lo anterior sería el comienzo, de allí se pasó a describir directamente el final de los ciclos de Luna para contarnos sobre su muerte: $y^{\prime} i l$, lo que ella tradujo como "morir", "estar muerto". Al transcurrir la Luna por el cuarto y último ciclo de fases, se expresa que "Luna muere", que "Luna está muerto": Wel'a y'il. ${ }^{14}$ Respecto de las fases lunares, en lo escrito por Mashshnek (1973, p. 117) también puede leerse que en cuanto es pensado como hombre, cuando llega a viejo no puede caminar, y muere para luego resurgir. De esta forma, la autora refirió en primer lugar al tercer y cuarto ciclo de fases, para culminar la explicación con el primer ciclo de fases: el cuarto creciente con el resurgimiento de Luna. Otra referencia bibliográfica corresponde a Roberto LehmannNitsche (1924), quién expresó que la Luna creciente era un buen momento para preparar bebida fermentada o para emprender la guerra. Métraux (1939, p. 9), por su parte, agregó que también era un buen momento para bailar al ritmo de los tambores. En cambio, según Lehmann-Nitsche (1924) mientras Luna está "muerto" no sería un buen tiempo para emprender ninguna actividad. Pérez Diez (1974, p.114) tradujo los términos wichís que nombran las fases lunares, asociando dos de ellas con los movimientos de la Luna en el cielo:15 "Luna recién sale", "néchie inúpa", ${ }^{16}$ y "Luna recién está en el medio", "Welá ihí lechó-wej". ${ }^{17}$ Los otros dos términos que transcribió refieren a situaciones distintas, el crecimiento de la Luna "Wéla néchie talók", expresión que significa "Luna está grande" o "recién es adulto", "recién es mayor"18 y la

\footnotetext{
14 "Y'il: enfermo; muerto" (Lunt, 2016, p. 116).

${ }^{15}$ Califano (1974: 48) también hace referencia al itinerario que seguiría Luna en el cielo, que sería el mismo camino marcado por el sol por arriba y debajo de la tierra.

16 "Nech'e: ya recién (más recién que 'ne'), nuevo" (Lunt, 2016, p. 65). "Nuphä: salir" (Lunt, 2016, p. 67).

17 Ihi: estar, existir, estar en (Lunt 2016: 40). -wej: cueva de animal (2016: 102).

18 Estas dos últimas traducciones fueron facilitada por Rodrigo
}

última sentencia que refiere a su muerte "Wéla yíl". Acerca de los ciclos lunares, John Palmer (2005) ofreció interpretaciones de mitos sobre Luna que logran asociar las fases lunares con los ritmos sociales. En uno de los mitos que transcribe John Palmer (2005, pp. 265-266) bajo el título: "la Luna y su bisnieta", describe el recorrido que hace Luna entre un poblado situado en el oriente y otro en el occidente (aguas arriba y aguas abajo, si tomamos como eje el río) para estar con su bisnieta. Al regreso de ambos se produce la violación de la nieta por el bisabuelo y la posterior menarca de la niña, remitiendo así al ciclo lunar completo y asociándolo con el ciclo femenino. También refiere a los ciclos lunares el mito que cuanta sobre la ejecución del tambor (pim pim) por parte de Luna. Al describir su forma de ejecución, el mito remite a la debilidad o fortaleza de Luna, dando así cuenta de cada una de las fases de la Luna que son relevantes para los wichí (Palmer, 2005, pp. 267-268). En el mito recabado por Celia Mashnshnek (1973, pp. 148-149), Luna era un hombre que mientras vivía en la tierra había causado la muerte a cinco mujeres debido a las grandes dimensiones de su miembro viril, sólo con la sexta (su esposa), supo cómo no lastimarla. Las familias de las jóvenes difuntas decidieron matar a Luna y luego de varios intentos fallidos lograron asesinarlo envenenándolo con una comida que le prepararon. Cuando estaba por morir, llegó a decirle a su esposa que cuando él regresara le tirara agua, porque iba a volver alumbrando. La mujer así lo hizo y logró apagarlo. Este hecho aconteció luego de tres días del entierro de Wel'a. Así es como siempre Luna desaparece y vuelve a aparecer alumbrando como Luna creciente. ${ }^{19}$ Luna es considerado el responsable de la menarca y por ello los ciclos menstruales y lunares están sincronizados (Barúa, 2001). De hecho, a la menstruación también se la llama "Wela walej", término que Pérez Diez (1974, pp. 114-115) traduce como "coito de Luna". Montani (2017, 352) también registra que "Walej cheyä" es una de las formas de llamar a la Luna llena y que esta designación significa literalmente "copula con la nieta", lo que nos remite al mito en el que Luna viola a su bisnieta, mito citado tanto en la compilación de Wilbert y Simoneau (1982, pp. 81-84) como por Palmer (2005, pp. 96-97, 265-268). Por otro lado, al igual que en otros grupos chaqueños, se considera que cualquier estrella que esté cerca de Luna es su esposa. Mario Califano (1974, pp. 48-49) registró que tanto Luna como Sol son habitantes del cielo y tendrían sus casas allí: Sol la tendría hacia el norte "lugar del que sopla viento norte" y Luna hacia el sur "lugar del viento sur o en dirección sur". De ahí las características térmicas de los lugares por los que sopla el viento: en el norte mora el calor "tajwoki" característico de Sol y hacia el sur el frío "fwyét" asociado con Luna (Califano, 1974, pp. 48-49). Luna también se relaciona con Sol al ser visto aquel como el hermano menor de éste y también como un personaje mucho más torpe y débil.

\footnotetext{
Montani.

19 Este mito también puede ser leído en un artículo publicado por Califano (1974, p. 48)
} 


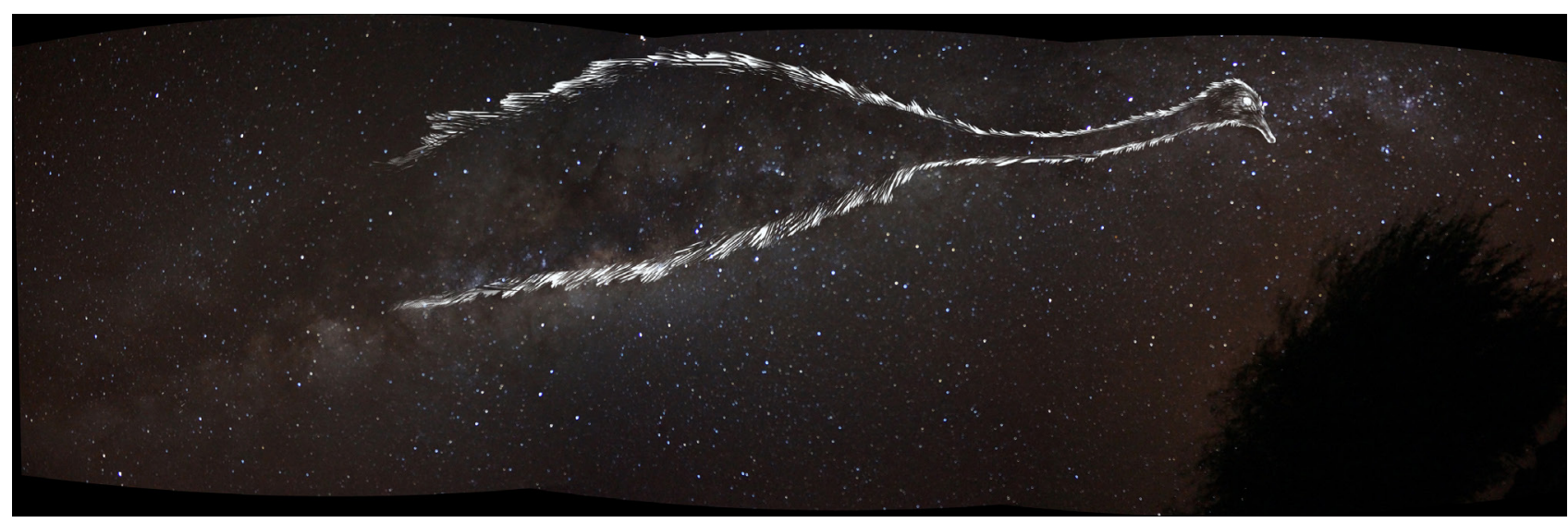

Figura 1. Wonlhoj, el Suri celestial. Representación gráfica del suri en la Vía Láctea

Figure 1. Wonlhoj, the celestial Suri (Rhea Americana). Graphic representation of the Suri in the Milky Way.

Sobre Sol (Fwala) nos explicaron que salía y se ocultaba por una cueva. De este modo, por lo menos una de las formas de llamar al naciente $u$ horizonte este y al poniente u horizonte oeste está relacionado con el Sol, siendo sus nombre Fwala pojthi y fwala weje respectivamente. Se le daría el nombre pojthi, ${ }^{20}$ porque por el horizonte este sale el Sol, y weje $e^{21}$ porque por el horizonte oeste se esconde. Respecto de estos términos, Montani (2017, p. 52) propone traducir "Ijwala-wej" por "trasero del sol" u "orificio del sol" para naciente y para el poniente propone interpretar los términos "ljwala yuy" como "el sol se mete". Siguiendo al mismo autor vemos que los otros dos nombres para oeste y este tienen como referencia el curso de los ríos: "río arriba": tewokw iphä y "río abajo": tewokw ichä, respectivamente. (Montani, 2017, p. 52). Respecto de los otros espacios cardinales $u$ horizontes, al Norte lo transcribieron como "fwfwñhi" y al Sur como "tapiñhi". ${ }^{22}$

Las estrellas son llamadas genéricamente katetsel (sing. kates), pero hay algunas que tienen nombres que las distinguen. A pesar de ser un planeta, desde la tierra y a ojo desnudo a Venus se lo puede observar como una estrella muy grande y los wichís la consideran así, como una estrella muy grande. Durante su visibilidad matutina y vespertina suele recibir nombres distintos puesto que muchas veces suele interpretarse que son dos estrellas distintas. El nombre brindado aquí es el de Fwalawo para el lucero matutino y Hunajwu para el vespertino. A diferencia de lo desarrollado en bibliografía (Braunstein, 1989; Lehmann-Nitsche, 1924), hasta el momento aquí no se ha podido constatar la relación entre Venus y el nombre Kates-Lhukwetaj. Otra de las situaciones que aún

\footnotetext{
20 -pofwohi: abierto; pofwthi: abrir cortando por el medio (Lunt 2016: 73)

21 -wej: sd. trasero, cueva de animal (Lunt 2016: 102).

22 Montani (2017, p. 52) da el mismo término para el sur, que se corresponde con el viento que va por esa dirección, mientras que para el norte da otro nombre: tahohi, el cual también corresponde al viento que va por esa dirección.
}

queda por indagar es si el lucero matutino y vespertino es reconocido como el mismo objeto celeste; situación que sí se da en, por lo menos, dos grupos orientales wichís (Braunstein, 1989, p. 46).

Como expresamos más arriba, el Suri es Ilamado Wänlhäj y es dibujado como un asterismo en las partes oscuras de la Vía Láctea. ${ }^{23}$ En la descripción que nos hicieron se le marca desde su cabeza (Ihetek), que se la identifica en la nebulosa oscura que está próxima a la Cruz del Sur y que es conocida como la bolsa de carbón. El cuello (lawu) se sigue dibujando en la mancha oscura de la Vía Láctea que continúa desde la anterior, pasa por Alfa y Beta de Centauro, se prosigue dibujando el lomo por la misma mancha oscura que es atravesada por la cola ${ }^{24}$ de la constelación de escorpio hasta pasar en medio de las constelaciones de Ofiuco y Sagitario ${ }^{25}$.

Las Pléyades, Ilamadas Pätselhay, fueron reconocidas, pero hasta ahora no se asoció ninguna historia a este asterismo del que se ha escrito bastante en la literatura etnográfica (vide infra). Las Nubes de Magallanes son identificadas como pozos de agua ${ }^{26}$ y Lehmann-Nitsche (1924, p. 265) registra para ellas el nombre "tsātaj", que traduce como terrón. La Cruz también es identificada en el cielo con ese mismo nombre y con las mismas estrellas

\footnotetext{
23 En una publicación reciente, Dasso $(2018$, p. 144) brinda una descripción diferente para el Suri o Wänlhäj. En la versión recogida por ella, el ojo del suri está representado por la estrella Alfa de la constelación de la Cruz del Sur (HIP 60718), y su cuello y cuerpo son marcados por fuera de la Vía Láctea, donde sólo se apoyan las patas. Esta descripción corresponde a comunidades wichís de la antigua misión Nueva Pompeya, en la provincia argentina de Chaco.

24 Para la correcta descripción de las estrellas referidas durante todo el artículo utilizaremos el catálogo brindado por el satélite Hipparcos. Estrellas nombradas: HIP. 86228, HIP. 84143, HIP. 82729, HIP. 82514 Y HIP. 82396.

${ }^{25}$ Estrellas de referencia: HIP. 85755 e HIP. 88635.

${ }^{26}$ Sin embargo el nombre que me brindaron para este asterismo fue Chowej y no lapek, éste último sí puede traducirse como pozo de agua. "Chowej: pozo, pl. chowhay" (Lunt, 2016, p. 22).
} 


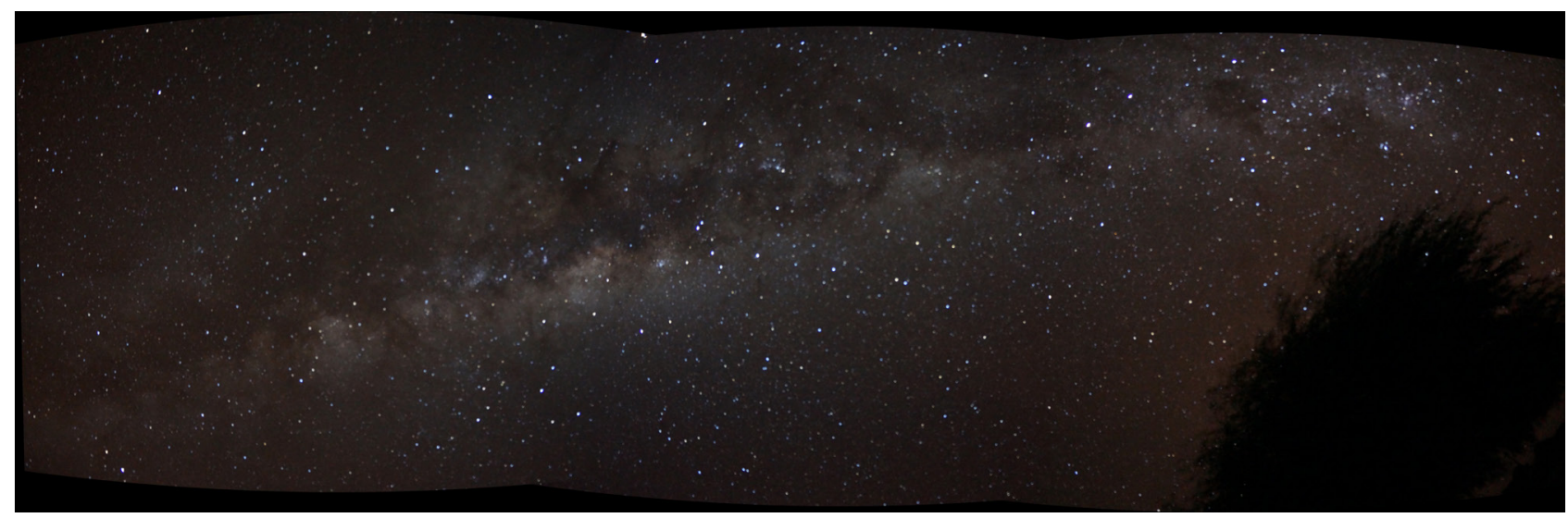

Figura 2: Wonlhoj, el Suri celestial (Vía Láctea)

Figure 2. Wonlhoj, the celestial Suri (Milky Way).

que forman la Cruz del Sur, pero no se la relacionó con ninguna narración o mito.

Uno de los asterismos que no tiene nombre en idioma wichí y hasta ahora no lo hemos encontrado en la literatura etnográfica sobre el grupo es la Cometa. Cuando consultamos sobre la razón de que no tuviera nombre en wichí, nos respondió: "No, la Cometa no tiene [nombre en idioma]. No, no tiene en idioma, porque la gente compara con el barrilete, la cometa". La cola de la cometa empieza en Alfa de Corona Borealis, traspasa todo Hércules por la serpiente; está a la derecha de Arturo. ${ }^{27}$

\section{Asterismos presentes en la bibliografía}

Hay asterismos y constelaciones que al día de hoy no nos han descripto pero están presentes en la bibliografía, entre ellos están algunos de los referidos por LehmannNitsche (1924). Estas diferencias nos hablan tanto de la gran variación dialectal y cultural que tienen los wichís a lo largo de su territorio, como de los cambios paulatinos que pueden haber sucedido con el transcurso del tiempo en las lecturas celestes. El asterismo formado por las estrellas Zeta 1 y Zeta 2 de la constelación de Escorpio no nos ha sido nombrado ni señalado. En cambio, en la literatura estas estrellas representarían a dos hermanos (Lehmann-Nitsche, 1924, p. 259), en tanto que Alfa 1 y Alfa 2 de Sagitario representarían a los padres de los niños (Braunstein, 1989, p. 47). Sobre el ave mítica relacionada con la lluvia y nombrada como el Gran Yulo, que según Lehmann-Nitsche (1924, pp. 261-265) estaría formado por Las Pléyades, Las Híades y el Cinturón de Orión, que serían respectivamente la cabeza, el cuerpo y las

\footnotetext{
27 El asterismo llamado la Cometa está formado por HIP 76267, HIP 72105, HIP 71284, HIP 70892, HIP 71075, HIP 73555, HIP 74666, HIP 75695 y la cola de la Cometa comienza a partir de HIP 76267 para continuar en HIP76952, HIP77512, HIP 78159, HIP 78493, HIP 80181, HIP 81693, HIP 912626, HIP 92420, HIP 91883, HIP 80816, finalizando en HIP 79593.
}

piernas, hasta ahora no nos fue descripta. Tampoco nos mencionaron al Cinturón de Orión como la extremidad inferior de Potselhay (cf. Braunstein, 1989, p. 58). María Cristina Dasso (2018, pp. 157-159) hace referencia a un Cuis (W'iyes), que es un asterismo que está situado entre Carina, Vela y Centauro, y también describe el Ala de Pato, pero hasta ahora no nos han hecho referencia sobre ellos. También se trazaría en el cielo wichí a un suri hembra y sus pichones, al unir estrellas de la constelación de Escorpio y Ara (Lehmann-Nitsche, 1924, p. 261); pero esta constelación tampoco nos ha sido mostrada ni nombrada. Hasta el momento, tampoco se trazó en La Vía Láctea un "Camino" (Lehmann-Nitsche, 1924, p. 265) y/o un "Río" (Braunstein, 1989, p. 59). Para este mismo asterismo Métraux (1935, p. 63) explica que se trata de las cenizas y el humo que quedaron en el firmamento luego de que se provocara un incendio en una porción del cielo para hacer un camino. Otra de las asociaciones con la Vía Láctea que hasta ahora no hemos relevado es la de ser un lugar por el que transitan las almas en sus vuelos extáticos (Braunstein, 1989, pp. 58-59). Aunque la constelación de la Cruz, tanto como Las Nubes de Magallanes, las Pléyades y el lucero, son reconocidas en el cielo y nombradas en wichí por nuestra colaboradora, no se les adjudicó ninguna narración o mito.

Otra situación, hasta ahora ausente en nuestros relatos, es que los wichís consideran que los excrementos de las estrellas pueden ser hallados en la tierra, siendo la evidencia unos hongos que pueden encontrarse en el monte (Suarez, 2014, p. 76). Montani (2017, p. 179) refirió a las estrellas fugaces como potaj ahat, unos diablos que se dirigen a las tumbas para comerse a quienes han fallecido recientemente. También son pensadas como las almas de los que están por morir y se van con los ahat/hais (Terán, 1999, p. 32, en Montani, 2019, p. 179). En esta misma tesitura también se explica que son las almas de chamanes poderosos que al estar por fallecer han de caer ruidosamente sobre una palmera (Braunstein, 1989, p. 59). En la bibliografía también se ha dejado constancia de la presencia en el cielo de un lugar de juego de los conejos (que entre los tobas del oeste formoseño se asocia a las Nubes de Magallanes) y de la presencia del Señor de los Tatúes (Métraux, 1935, p. 63). 


\section{Interpretaciones}

Respecto de Pätselhay, las Pléyades, en la bibliografía abundan las interpretaciones sobre las narraciones que se le asocian, las cuales suelen ser interesantes ejemplos de la relación entre el cielo, sus asterismos y la sociedad que los lee. Barúa (2001, pp. 37-39) brindó una lectura que enlaza a Potselhay con la fertilidad y los nuevos ciclos de abundancia. En este relato se sugirió que la función del asterismo es fecundar las semillas terrestres para que ellas crezcan bien y que también en el monte haya abundancia de alimentos. También describió que los chamanes del grupo tenían incidencia en que el desarrollo del ciclo se diera correctamente. Esta narración podría enlazarse con aquellas que hablan de su aparición como un importante marcador del comienzo del ciclo anual, cosa que también sucede entre los tobas del oeste formoseño. En este grupo toba, así como entre los pilagás, al asterismo asociado a las Pléyades se lo conoce con otro nombre (Dapichi), pero también se lo relaciona con el comienzo del año, teniendo a su cargo la fructificación del monte y siendo el responsable del buen crecimiento de las semillas. Aunque actualmente los wichís suelen consignar el comienzo del año a fines de diciembre, los más ancianos expresaban que el comienzo del nuevo ciclo anual se daba en el momento en el que las Pléyades aparecían en el horizonte oriental un poco antes del amanecer, durante la salida Helíaca de las Pléyades en el hemisferio sur (Alvarsson, 1988 , p. 58). Por otro lado, Palmer (2005, pp. 285-286) también explica que los chamanes dicen que al aparecer Pätselhay (las Pléyades), hacen un ruido de niños jugando. Hay otros mitos que explican el origen de este cúmulo estelar, uno de ellos cuenta que son unos cazadores que se quedaron allí por la acción de un anciano a quién habían irritado (Métraux, 1939, p. 9). Otro mito que habla sobre su origen ha sido anotado y comentado por Palmer (2005, pp. 277-287) bajo el título: El origen de las Pléyades. Allí, en principio, reflexionaría sobre el parentesco, aludiendo a la posición muy desfavorable del yerno frente a su suegro en un grupo familiar como consecuencia de una pauta de residencia postmarital uxorilocal. Este mito también remitiría a la prohibición de contraer matrimonio con una hermana clasificatoria. Así, Pätselhay (las Pléyades) codifica simbólicamente, en el asterismo y en el mito que se le está asociado, unas prescripciones sociales y denuncia la "falta de reciprocidad por parte de los dadores de esposa en una alianza afinal" (Palmer, 2005, p. 141). ${ }^{28}$ En este último caso, Las Pléyades representan a unas corzuelas que escapan al cielo para evitar que su abuelo las mate. ${ }^{29}$ Esta persecución deviene

\footnotetext{
${ }^{28}$ Esta interpretación ligada al parentesco es trabajada en detalle por Palmer 2005, pp. 139-141, 277-287.

${ }^{29}$ Hay muchas versiones de cada uno de los mitos a los que aquí nos
}

Figura 3. La Cometa

Figure 3. The kite.

luego de que el abuelo de estas corzuelas (el jaguar) hubiera asesinado a su yerno (una corzuela que era el esposo de la hija de jaguar). En venganza, los hijos de la corzuela y nietos del jaguar, le dan de comer a su abuelo jaguar la carne de sus tíos maternos (los propios hijos del jaguar). Cuando el jaguar se da cuenta de esta situación, corre a sus nietos, pero ellos logran huir y se quedan en el cielo, donde forman a las Pléyades. Un relato recabado por Eugenia Suarez (2012, p. 155) muestra otra característica de las Pléyades que da cuenta de una prohibición también vigente entre los tobas del oeste formoseño y se resume en los peligros que acarrea mirar el cielo. Así se advierte que aquellos niños que miren mucho a Pätselhay (Pléyades) pueden quedarse sin su lahusek ("voluntad", "alma") puesto que el asterismo se las podría robar. ${ }^{30}$

Así como Pätselhay podía robar el lahusek de los hombres o niños, generando una enfermedad a quien le suceda (Califano, 1974, p. 49; Suarez, 2012, p. 155), otro personaje celeste que podía hacerlo es el lucero (Venus), en tanto represente a Kates-Lhukwetaj. Uno de los mitos relacionados con este objeto celeste cuenta que Kates-Lhukwetaj, la Mujer Estrella, encandila a los jóvenes que la miren, dejándolos sin su lahusek, quienes

referimos y cada una de ellas depende mucho del contexto en el que sean narradas, pese a que todas sean consideradas parte de un mismo relato. El sentido del mito sería más claro si sobre cada uno de ellos se trataran de relacionar analíticamente el mayor número posible de variantes. Pero dado el límite de espacio del presente escrito, aquí se presentan versiones esquemáticas.

${ }^{30}$ Hasta hoy no se nos habló sobre ninguna de estas características de Pätselhay, pero hay que recordar que este trabajo es sólo un primer acercamiento al tema. 
sólo podrán recuperarlo mediante la intervención de un chamán. Los afectados por esta dolencia sufrían de un gran frío, adelgazaban mucho y casi no hablaban; en algunos casos el frío era de tal magnitud que intentaban introducir pequeñas brazas encendidas en su boca (Califano, 1974, p. 50). Estos efectos son propios de alguien que ha mirado mucho al cielo, pareciendo quedar al borde de la locura. El mito relacionado con este objeto celeste está ampliamente extendido en la zona chaqueña, pero también hay más versiones distribuidas en toda América (cf. Blixen, 1991; Gómez, 2007). En el caso wichí, hay registradas bastantes versiones del mito de la Mujer Estrella; aquí sólo contaremos a grandes rasgos su argumento. Existía un hombre que era muy feo, al que ninguna mujer del poblado se acercaba y por lo tanto no podía tener pareja. Este hombre se enamoró de una estrella quién finalmente bajó y estuvo con él. En algunas versiones la Mujer Estrella se queda un tiempo con él en la tierra, haciendo crecer frutos en momentos de carestía. Pero, finalmente, la Mujer Estrella regresó al cielo. El hombre desesperado logró seguirla y llegar hasta el cielo. Pero vivir en el cielo es algo que sólo pueden hacer los chamanes, por lo tanto el hombre resultó muerto por acercarse mucho al fuego, que como todo fuego celeste explota. En algunas de las versiones es el padre de la Mujer Estrella quién arroja al hombre al fuego (Dasso, 1989, p. 32; Palmer, 2005, pp. 294-295; Wilbert \& Simoneau, 1982 , p. 49) y en otras es él mismo quien no le hace caso a su esposa y al atizar el fuego éste explotó y el hombre resultó muerto. Para que sus parientes supieran que él había muerto envían sus huesos a la casa de su familia por medio de un ave: chestaj. En un análisis que trabajamos hace unos años (Gómez, 2009), barajamos una hipótesis en la que establecíamos que el mito pondría en escena los peligros que implica una alianza no determinada por las reglas de alianza establecidas y descriptas con distintos grados de precisión o desde diferentes ángulos por distintos autores (Alvarsson, 1988; Braunstein, 1983; Montani, 2017; Palmer, 2005). Por un lado, y al contrario de lo que se aconseja, fue el hombre quien eligió a la Mujer Estrella, éste se ofrece y va solo hasta la casa de ella, sin que ella lo llevara hasta allí. Esta situación deja al hombre en una posición muy desfavorable, quedando especialmente expuesto ante sus afines pero, sobre todo, a disposición de su suegro (kachoti). Por otro lado, se plantea una relación que está por fuera de los parámetros establecidos, una unión que es excesivamente exógama, que está por fuera de aquellos con quienes sí se debería haber establecido: los que han sido catalogado como "otros diferentes"; siguiendo de esa forma un principio de "afinidad serial" (Palmer, 2005, pp. 141-142). Esta norma de alianza la expresó Montani (2017, p. 94) de la siguiente forma: así como está prohibido casarse con parientes cercanos, también es costumbre evitar el casamiento con gente muy lejana o totalmente extraña, con aquellos que estén por fuera de quienes ya pueden ser catalogados como afines potenciales, personas con quienes sus parientes ya han trazado lazos de alianza.
La situación de alianza que plantea el mito de la mujer estrella está por fuera de lo recomendado, por lo tanto esta relación puede generar complicaciones, es peligrosa (Palmer, 2005, pp. 96-152) y esta peligrosidad es consecuentemente ratificada por el mito.

Por otro lado, en tanto que son considerados habitantes del ámbito celeste también debemos incluir en estas notas lo recabado por Mario Califano y María Cristina Dasso (1988), nos referimos a otros dos seres poderosos que habitan el cielo wichí: "Itox pahla" e "Innó pahla", que son personajes del tiempo mítico. A estos personajes celestes se suman aquellos que los wichís tomaron de las religiones cristianas, que van desde Jesucristo hasta los sacerdotes o religiosas que mueren (Califano \& Dasso, 1988, p. 41). Como expresa Montani (2017, p. 112), las creencias wichís también se han visto modificadas con la introducción de las traducciones bíblicas. Las enseñanzas de los anglicanos se han amalgamado con las creencias tradicionales wichís. Así es que se pueden encontrar lecturas sobre el cielo donde, por sobre todos los seres celestes, ahora está Dios. En un mapa cosmográfico brindado por el mismo autor (Montani, 2017, p. 121) se explica que entre los estratos superiores y la tierra hay puertas de acceso; además, por debajo de Dios que está arriba de todo en el estrato superior, habría "diablos" que viven en túneles. Un elemento nuevo sería que por debajo de esos "diablos" se entiende que están las "almas buenas", situación que antes no estaba contemplada dado que toda persona se convertiría en algún momento en ahat y su lugar no era el cielo (como aquí se propone), sino el inframundo. Ahora, a partir de la evangelización, estas "almas buenas" pueden morar en el cielo.

\section{Consideraciones finales}

De alguna manera, y tomando lo que propuso Montani (2017) para los wichís, se comienza a divisar el cromatismo que se da entre los distintos grupos wichís también expresándose a través de los asterismos e interpretaciones celestes. Por otro lado, así como con el correr de los años y con la influencia de otras religiones cristianas se agregaron otras lecturas del cosmos, también parecen suceder cambios en las lecturas del cielo que no implican directamente las nuevas enseñanzas religiosas. Por ejemplo, agregar el asterismo de la Cometa al cielo wichí hace pensar en nuevas lecturas que se entretejen a la vida cotidiana más reciente.

A lo largo del artículo sí hemos podido ver que Luna, Wel'a, es pensado de forma unánime en masculino, un personaje celeste que se relaciona a partir de varios relatos con la menstruación y la menarquía. Luna también se ve asociado a períodos de tiempo acompasados a los ciclos femeninos, dando pautas de un ciclo concreto y mensurable en la mujer, pero también por la trayectoria y formas cambiantes de la Luna, lo que podría asociarse al mes sinódico. Hoy día la asimilación entre Luna y 
el mes marcado por el calendario occidental parece ser indisoluble. Por otro lado, sobre Sol, Fwala, no parece darse una lectura unánime en cuanto al género que representa; por lo menos al día de hoy ello no es expresado con tanta claridad. En el trabajo de campo la relación entre el Sol y la forma de nombrar los momentos del día aún no ha sido trabajada con precisión; sin embargo, parece ser una hipótesis plausible al tomar en cuenta las formas de nombrar algunas de las partes en las que dividen el día. ${ }^{31}$ Del mismo modo, resulta sugestiva la forma de nombrar al reloj32: "fwalacha" (Lunt, 2016, p. 29) donde ocurre la palabra sol y "-cha", que estaría indicando que se habla de un instrumento de la primera parte del término, en este caso sol: fwala. ${ }^{33}$ Una hipótesis preliminar de trabajo sobre la palabra (reloj) fwalacha, podría decir que además de ocurrir en ella la palabra sol: fwala, también está la palabra -cha, que según el diccionario (Lunt, 2016, p. 19) indica "cosa, herramienta, diseño". Con lo cual, evidentemente, podría pensarse como la herramienta o instrumento del sol. Sin embargo, también podría estar refiriendo al diseño que el sol dibuja en el cielo con su recorrido. Del mismo modo, también podría pensarse que estaría dando cuenta del diseño que el sol hace en la tierra con las sombras que provoca. Es justamente mediante las distintas formas y direcciones que tengan las sombras "diseñadas" por la luz del sol que también se puede saber en qué momento del día se está, esos "diseños" serían un indicador del instante del día u "hora" y oficiaría de reloj. ${ }^{34}$

Por otra parte, un cotejo inicial nos permite ver que hay asterismos que muy difícilmente no estén presentes cuando se trabaje sobre el cielo, pensamos en las Pätselhay (Pléyades), Wänlhäj (Suri) y Chuwej (las Nubes de Magallanes). En los relatos trabajados aquí, el lucero y las Pléyades siguen siendo igual de importantes, pero los relatos que se le asocian no están tan claramente vigentes. Los únicos relatos míticos relacionados al cielo que sí estaban vigentes y anotamos más arriba fueron el que relata la inversión de lugar entre el cielo y la tierra, y el que cuenta sobre el advenimiento de las mujeres. Por otro lado, al contrario de lo que esperábamos encontrar, el asterismo que primero asociaron a la llegada del frío fue Wänlhäj (Suri) y no Pätselhay (Pléyades). Con el correr del tiempo, seguramente lograremos dar con más ancianos que recuerden sobre el cielo. En el presente trabajo se intentó dejar plasmado, tanto lo que continúa en vigencia, así como las ausencias y los cambios, que estimamos son tan significativos como las continuidades. Ahora, el cambio de ciclo anual se asocia con el período

\footnotetext{
31 "Tojchä ifwala: madrugada", "fwala inuphä: el amanecer", "nech'e fwala: la mañana", "fwala tott'aho lew'et: mediodía", "fwala itilhakhi: la tarde, entre las 13 y las15 horas", "fwala y'uy / ifwala wej ichät: ocaso" (Lunt, 2016, p. 29).

32 También se puede decir "reloj pulsera: kaijwala(s)", donde también ocurre la palabra Sol: "ijwala" (Montani, 2017, p. 511).

33 Cf. Montani, 2017, pp. 282-283.

${ }^{34}$ Esta forma de indicar el día ha sido profundamente trabajada entre los tobas del oeste formoseño (Cf. Gómez \& Carpio, 2018).
}

de abundancia, durante el mes de diciembre y ya no con la reaparición de las Pléyades. A los meses le dicen lunas, pero la práctica cotidiana de la ciudad parece usarse más como sinónimo de mes que refiriendo el período sinódico lunar. Esta situación iría en concordancia a los ritmos propuestos por la vida en la ciudad, que se imponen a las lecturas tradicionales aunque sin Ilegar a anularlas. Lo expresado hasta ahora parece referir a como se encarnan estos nuevos ritmos impuestos, lo que parece hacerse a partir de su propia cosmovisión, sin perderse o tomar sin más el ritmo temporal que impone la vida cotidiana en la ciudad. Pareciera que las miradas sobre el cosmos pueden ir cambiando en la medida que los propios wichís lo consideran necesario: nuevos elementos se incorporan, otros se transforman y claramente el cielo como parte de la cosmovisión también cambia y se va modificando.

Buenos Aires, 31 de julio del 2019.

\section{Agradecimientos}

Quiero expresar mi agradecimiento al Dr. Ignacio Ranea Sandoval, quien desinteresadamente facilitó la fotografía de la Vía Láctea tomada por él en el invierno del año 2014 en el oeste de la Provincia de Formosa (Argentina). La misma fue tomada en el marco del proyecto de Extensión Universitaria "Astronomía en el oeste formoseño. Una aproximación a partir de la astronomía cultural", que fue financiado por la Facultad de Ciencias Astronómicas y Geofísicas de la Universidad Nacional de la Plata. Sobre esta fotografía hemos podido trazar el asterismo llamado Wän/häj (Suri). También agradezco muy especialmente al Lic. Sixto Giménez Benítez por la colaboración en el trazado del asterismo wichí "La Cometa" en el Software Stellarium (0.16.0), que ha sido utilizado como base para su diseño en este artículo. Las representaciones gráficas sobre los asterismos Wän/häj (Suri) y La Cometa fueron realizadas por el ilustrador Lic. Diego Alterleib. Para finalizar, debo agradecer a la Dra. Zelda Franceschi y al Dr. Rodrigo Montani por las observaciones y comentarios realizados a una primera versión de este trabajo.

\section{Bibliografía}

Alvarsson, J.-Å. (1983). The origin of Mataco women. Latin American Indian Literatures, 2, 167-169.

Alvarsson, J.- $\AA$. (1988). The Mataco of the Gran Chaco: An Ethnographic Account of Changeand Continuity in Mataco Socio-Economic Organization. Series in Cultural Anthropology Vol.11. Uppsala: Acta Universitatis Upsaliensis.

Arenas, P. (2003). Etnografía y Alimentación entre los Toba-Ñachilamole\#ek y Wichí-Lhuku'tas del Chaco Central (Argentina). Buenos Aires: Dunken.

Aveni, A. (1991). Observadores del Cielo en el México 
Antiguo. México: Fondo de Cultura Económica.

Barúa, G. (2001). Semillas de estrellas. Los nombres entre los wichí. Buenos Aires: Dunken.

Belmonte Avilés, J. A. (1999). Las Leyes del Cielo. Astronomía y Civilizaciones antiguas. Madrid: Editorial Temas de Hoy.

Blixen, O. (1991). La mujer estrella. Análisis de un mito sudamericano. MOANA 4(1), 1-27.

Braunstein, J. (1974). Dominios y jerarquías en la cosmovisión de los matacos tewokleley. Scripta Ethnologica, 2(2), 7-30.

Braunstein, J. (1983). Algunos rasgos de la organización social de los indígenas del Gran Chaco. Serie Trabajos de Etnología Nro. 2. Buenos Aires: Facultad de Filosofía y Letras.

Braunstein, J. (1989). Astronomía mataca. Revisión crítica de la Mitología Sudamericana $V$ de Roberto LehmannNitsche. Scripta Ethnologica, Supplementa 9, 43-60.

Califano, M. (1974). El concepto de enfermedad y muerte entre los mataco costaneros. Scripta Ethnologica, 2(2), 33-73.

Califano, M. y Dasso, M. C. (1988). Diccionario referencial de teofanías wichí. Mitológicas 3, 37-46.

Dasso, M. C. (2018). La Mirada vigil. La percepción de la noche en el mundo humano. Archivos 16, 121-176.

Feinstein, A. (1969) Astronomía elemental. Buenos Aires: Kapeluz.

Fock, N. (1982). Introduction: History of the Mataco Folk Literature and Research. En Wilbert, J., Simoneau K. (Eds.). Folk Literature of the Mataco Indians. Los Ángeles: University of California, 1-33.

Gómez, C. P. (2007). Kates Lhukwetah: La representación social, material y estelar de la mujer entre los Wichí. Acta Americana 15(2), 97-115.

Gómez, C. P. (2009). Parentesco y representación estelar de la mujer entre los Wichí del Gran Chaco. En Actas del $V I^{\circ}$ Congreso de Americanistas (pp. 205-219). Buenos Aires: Dunken.

Gómez, C. P. y Carpio M. B. (2018). Ahewa Likíri: El reloj y la jornada entre los tobas del oeste fromoseño (Guaycurú-, Argentina). Espaço Amerindio, 12(1), 144-173.
Iwaniszewski, S. (1994). De la astroarqueología a la astronomía cultural. Trabajos de prehistoria, 51(2), 5-20.

Lehmann-Nitsche, R. (1924). La Astronomía de los Matacos. Revista del Museo de La Plata, 27, 253-265.

Lunt, R. (2016). Diccionario de la lengua wichí. Buenos Aires: Sociedad Bíblica Argentina.

Mashnshnek, C. O. (1973). Seres potentes y héroes míticos de los mataco del Chaco Central. Scripta Ethnologica, 1(1), 105-154.

Métraux, A. (1935). El universo y la naturaleza a través de las representaciones míticas de dos tribus salvajes de la Argentina. Sur, 10, 54-70.

Métraux, A. (1939). Myths and Tales of the Matako Indians (The Gran Chaco, Argentina). Ethnological Studies 9, 1-127

Montani, Rodrigo (2017) El mundo de las cosas entre los wichís del Gran Chaco: Un ensayo etnolingüístico. Colección Scripta Autochtona, 17. Cochabamba: Itinerarios

Palmer, J. (2005). La buena voluntad wichí: Una espiritualidad indígena. Formosa y Salta: APCD, CECAZO, EPRAZOL, FUNDAPAZ, Franciscanas Misioneras de María, Parroquia Nuestra Señora de la Merced, Tepeyac, Asociana.

Pérez Diez, A. (1974). Noticia sobre la concepción del ciclo anual entre los matacos del noreste de Salta. Scripta Ethnológica 2(1), 111-120.

Ruggles, C., Saunders N. (1993). The Study of Cultural Astronomy. En Ruggles C., Saunders N., (Eds.). Astronomies and Cultures. Nitwot: University Press of Colorado, 1-31.

Suárez, M. E. (2012). Espíritus vinculados con el bosque y sus plantas en el mundo de los wichís del chaco semiárido salteño, Argentina. En P. Arenas (Ed.), Etnobotánica en zonas áridas y semiáridas del conosur de Sudamérica (145-178). Buenos Aires: CEFYBO, CONICET.

Suárez, M. E. (2014). Etnobotánica wichí del bosque xerófito en el Chaco semiárido salteño. Don Torcuato: Autores de Argentina.

Wilbert J. y Simoneau K. (1982). Folk Literature of the Mataco Indians. Los Ángeles: UCLA Latin American Center Publications. 